\title{
Material Design for Application to High Performance LCDs.
}

\author{
Atsushi Kumano \\ Display Materials Laboratory, Fine Electronic Research Laboratories, JSR Corporation, \\ 100 Kawajiri-cho, Yokkaichi, Mie 510-8552, Japan
}

\begin{abstract}
Liquid Crystal Display(LCD) is a high performance tool for displaying many kinds of information. Application of $\mathrm{LCD}$ is diversifying to notebook PC, monitor display, TV and reflective type of PDA and personal handy phone. To achieve these performance for several applications of LCD, development of new materials has been investigated. Color photoresist is used for color filter production. Negative and positive type of transparent photoresist are applied for leveling and overcoating layer of LCD process , and further used for spacer materials. For the next generation of alignment layer; photo-alignment material is also investigated. Dimerization of chalcone moiety showed good alignment characteristics for liquid crystals and good LCD performance.
\end{abstract}

Keywords: color photoresist, negative-, positive photoresist, photo-alignment

\section{Introduction}

Liquid crystal display(LCD) has been started by a dynamic scattering mode of segmented display for a watch and calculator"). In 1986, STN-LCD has been investigated by using rubbed polyimide alignment layer. Monochromic STN-LCD has been changed to full-color display, and now active matrix type of TFT-LCD is a major display. LCD's market has been widely growing by application to a notebook PC recently. Nowadays, it shows various applications such as monitor display for PC, TV of large size of display and personal digital assistant(PDA), personal handy phone of small size display. There are many target performances which have been investigating for the wide angle viewing, high definition display and low consumption of energy by reflection type of $\mathrm{LCD}$. To achieve these performances of LCD, much efforts have been doing to develop new organic materials.
In this paper, color photoresist material, negative and positive type of transparent photoresist and photoalignment layer materials are introduced,
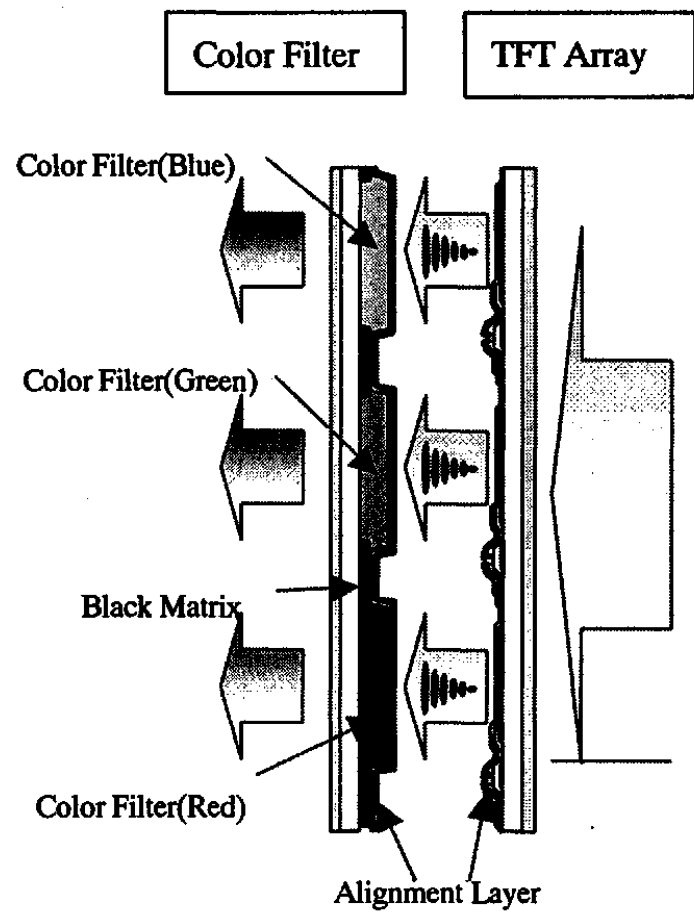

Fig. 1. Cross sectional view of LCD panel. 
which contribute to high performance of LCDs.

\section{Color photoresist for the color filter}

Color filter (CF) is an important component for color LCD. Pigment dispersed color photoresist method is a major procedure of CF production. In this section, color photoresist materials of red, green and blue pigment dispersion system are introduced. And also, black photoresist for black matrix is mentioned here.

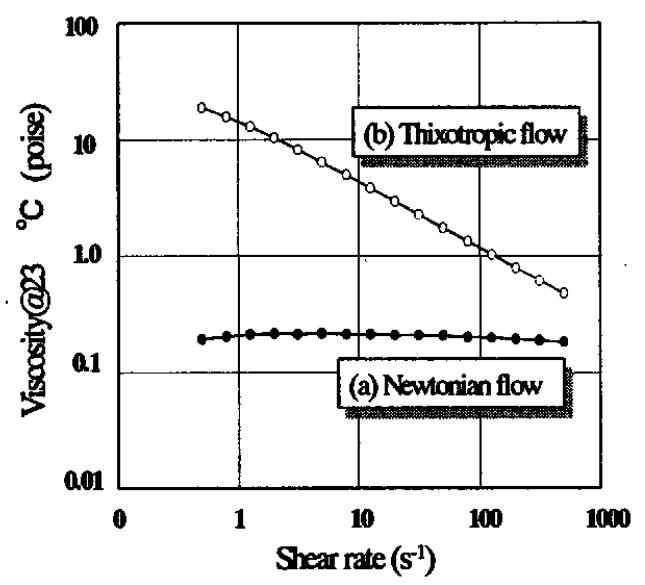

Fig. 2. Rheological characteristics of color photoresist.

Color photoresist is made from transparent photoresist dispersed ultra-fine particles of pigment, which pigment size is about $100 \mathrm{~nm}$ in diameter. It is coated on a glass plate and exposed by $i$-line of UV light, and developed by alkaline solution. This sequential process is repeated three times, and then CF is formed. As a material design of color resist, it is needed to keep a uniform coating. In LCD production, $550 \mathrm{~mm}$ $\mathrm{x} 650 \mathrm{~mm}$ or more larger size of glass plate is adopted, therefore Newtonian flow of color photoresist is needed for achieving a uniform thickness(Fig.2 (a)). Thixotropic flow of color photoresist shown in Fig. 2(b) shows large difference of film thickness distribution and then, leads to different color chromaticity on a same glass plate. Design for the photolithographic material is also difficult, because pigments absorb and diffract UV light. It is difficult to keep a same condition of photoreaction from top to bottom of color photoresist layer.

In recent year, $\mathrm{CF}$ is required a high transmittance and high chromaticity. To achieve this request, fine particle of pigment is selected in addition to a new pigment investigation. High transmittance of color photoresist is achieved by using a small particle size of pigment. For high chromaticity, high pigment content is needed, so that the selection of dispersants and chemical design for photoresist material having an affinity for pigment are very important. Fig. 3 shows a typical transmission spectra of red, green and blue of color photoresist. Pigment investigation, minimize of particle size of pigment and chemical design of photoresist have performed a high transmittance and high chromaticity of color filter. High resolution of color photoresist is also required for high quality and small pixel size of LCD.

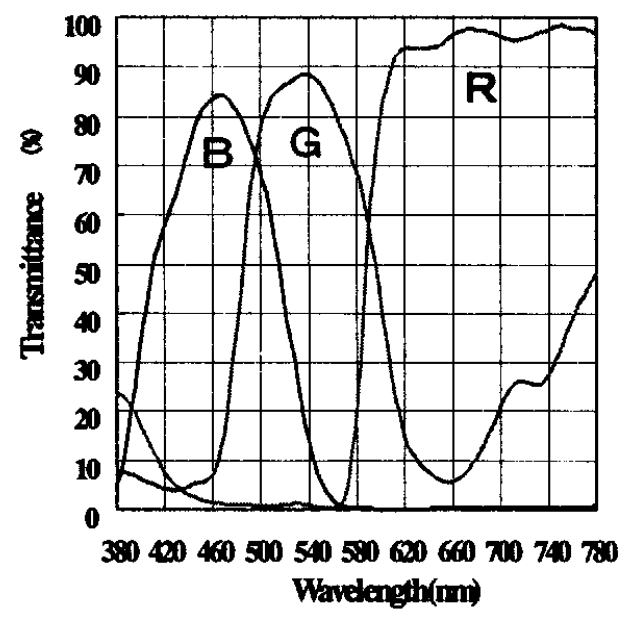

Fig. 3. Transmission spectra of red, green and blue color photoresist. 
From the requests of cost reduction, lowering reflection and industrial pollution problem, black color photoresist materials are investigated instead of chromium black matrix. Carbon black or mixed pigments of red, green and blue are used. Black photoresist will be required high optical density of $>3 / \mu \mathrm{m}$, high resolution of under $10 \mu \mathrm{m}$ and high electro-resistivity. Black photoresist has to achieve both high light shield characteristics and high photosensitivity.

\section{Negative type of photoactive material}

\section{for the spacer of LCD} (Photolithographic spacer)

The spacer material is adopted to keep a cell gap of LCD. Mono-dispersed fine particle of plastic or silica is used for it. These are sprayed on a base glass, so that particles have to exist on a pixel. Therefore a light leak or distortion of alignment of liquid crystals will be observed around particles. And also, it is difficult to spray uniformly in a large size of LCD panel and particles are moving by an oscillation, and then the uniformity of cell gap has been collapsed.

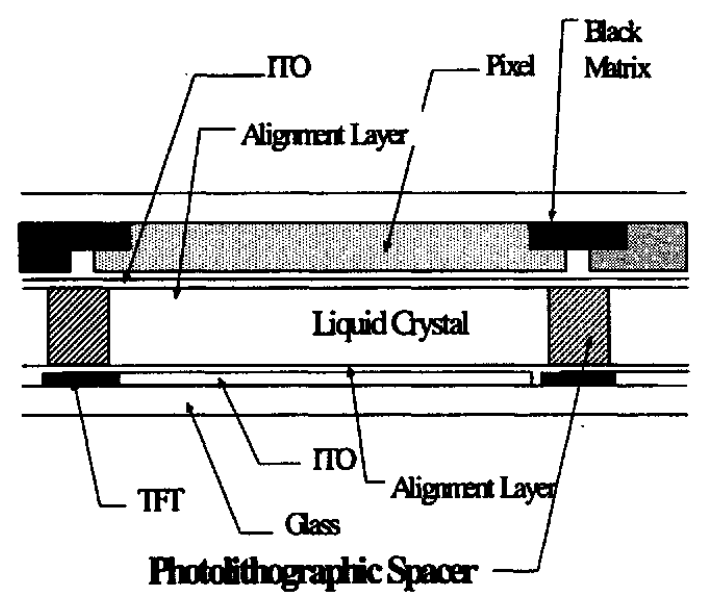

Fig. 4. Schematic illustration of photolithographic spacer in LCD cell.

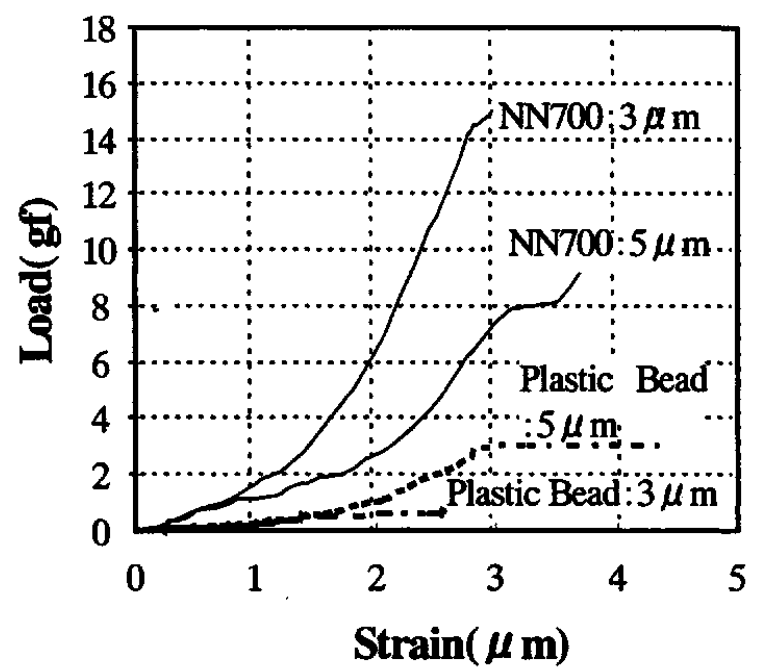

Fig. 5. Stress-Strain curve of NN700 and plastic bead.

Photoactive spacer materials (photolithographic spacer) are proposed to avoid these problems ${ }^{2}$. Negative type of photoresist material is coated on a base glass with uniform thickness, and designed size of pattern is formed by photolithographic method on a desired position(Fig.4). Resolution of photolithographic spacer is required about $10 \mu \mathrm{m}$ or less. It is also needed mechanical strength and recovery against an elastic strain. Fig. 5 shows stress-strain curves of photolithographic spacer, JSR NN700 and plastic bead with different size. NN700 shows a low strain under same stress condition.

Display performance is strongly depended on the uniformity of cell gap ${ }^{3)}$. Therefore, a height uniformity of photolithographic spacer is the most important. NN700 also showed a uniform coating of thickness and its height was 4.50 $\pm 0.04 \mu \mathrm{m}$. Patterned profiles of NN700 are shown in Fig. 6.

Photolithographic spacer is directly contact with liquid crystalline materials, so that the material design to avoid dissolution 
from photolithographic spacer has to be required.
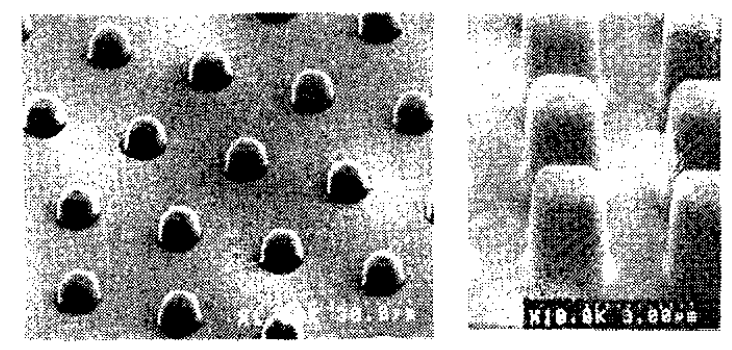

Masksize : $10 \mu \mathrm{msq}$
Coating thickness: $5 \mu \mathrm{m}$
Exposire $\quad$ : Aligner

$2 \mu \mathrm{msq}$

$26 \mu \mathrm{m}$

i-line stepper

Fig. 6. Pattern profile of photolithographic spacer material, NN700.

\section{Positive type of photoactive material} for the permanent layer inside LCD

Typical application of positive type of photoresist is for TFT formation processing. In this section, permanent type of photoactive materials will be mentioned. Important characteristics of these materials are both thermal crosslinking feture and high transparency ${ }^{4)}$. After photo-process, unexposed region is formed cross-linked structure thermally and then, works as a stable permanent cured layer. As a transparent material, post-exposure of light is needed. Residual photoactive compound in unexposed region is changed to acid type of compound and then, reacts with a base resin to form a cross-linking structure. These two processes make a photoactive material to apply a LCD panel as high reliable material.

Fig. 7 shows a post exposure dependence of transmission spectra of JSR PC403. Depending on the exposure energy, transmittance of PC403 is increased. PC403 shows over $90 \%$ of transmittance at $400 \mathrm{~nm}$ on $3 \mu \mathrm{m}$ of thickness.

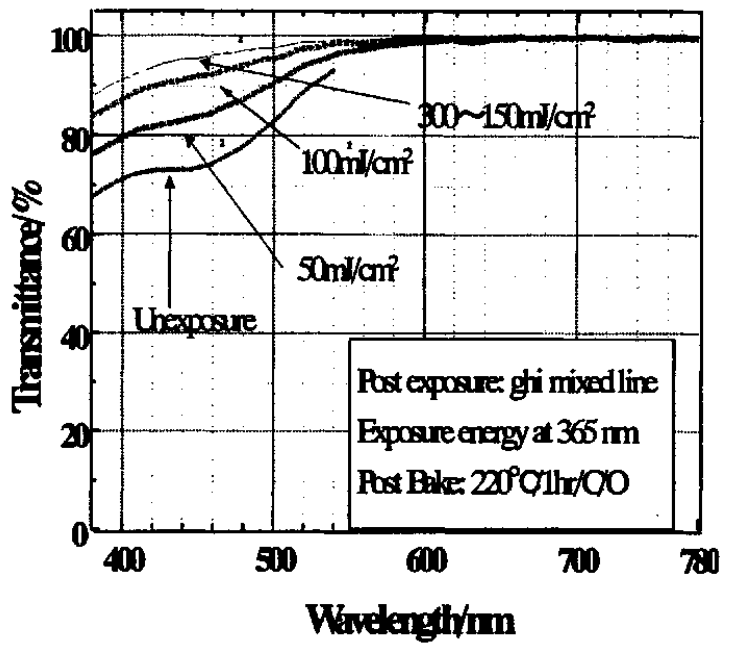

Fig. 7. Post exposure dependence of transmittance of PC403.

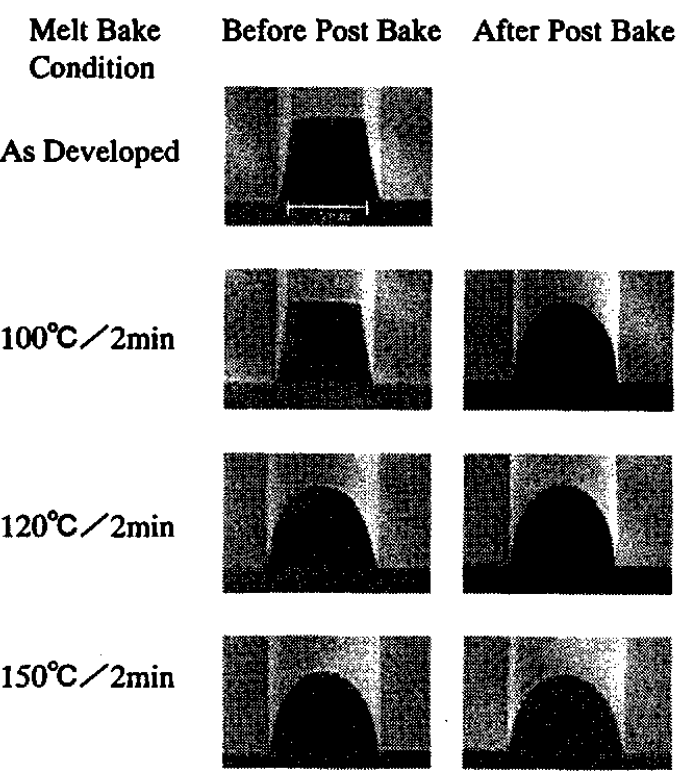

Fig. 8. Melt flow patterns of $\mathrm{PC} 403$ at several melt bake temperature.

Two kinds of the thermal baking processes are also important for PC403. One is a melt flow process(melt bake), in which the developed pattern of PC403 are formed by thermal process. And next, to finish a thermal cross-linking, PC403 is baked at around $220^{\circ} \mathrm{C}$ (post bake). Fig. 8 shows melt flow patterns at several melt bake 
temperatures. Desired pattern profile could be possible to control by selecting a melt bake temperature.

Positive type of photoactive material is applied to transparent leveling layer on TFT array ${ }^{5}$, in which it is needed to form a contact hole for electrical conduction. And also, this type of photoresist is applied to a rib formation for a panel of multi-domain vertical alignment ${ }^{6)}$. Typical characteristics of cured film of PC403 are shown in Table 1.

Table 1. Typical characteristics of cured PC403.

\begin{tabular}{ll}
\hline & \multicolumn{1}{c}{ PC403 } \\
\hline - Transparency $/ \%$ & $>93$ at $3 \mu \mathrm{m}$ \\
- Pencil hardness & $3-4 \mathrm{H}$ \\
- Refractive index & 1.55 at $632.8 \mathrm{~nm}$ \\
- Specific volume & \\
Resistance & $1.6 \times 10^{15} \Omega \cdot \mathrm{cm}$ \\
- Dielectric constant & 3.3 at $\mathrm{f}=100 \mathrm{kz}$ \\
\hline
\end{tabular}

\section{Photo-alignment material}

To align the liquid crystalline molecules on the plane uniformly, alignment layer is needed and it's surface has to be rubbed by the cloth. Polyimide is a major alignment material in the LCD production. However, by rubbing process, there are some problems such as dust generation, static electricity and difficulty of process control for large size panel. To improve these problems, several photo-alignment methods have been investigated.

Polymer with a 4-chalconyl group as a side chain has developed ${ }^{7}$. Chalconyl group dimerizes by relatively longer wavelength of UV light around $365 \mathrm{~nm}$. Photoreaction at longer wavelength is good for chemical stability, because there are no degradation or oxidation reaction.

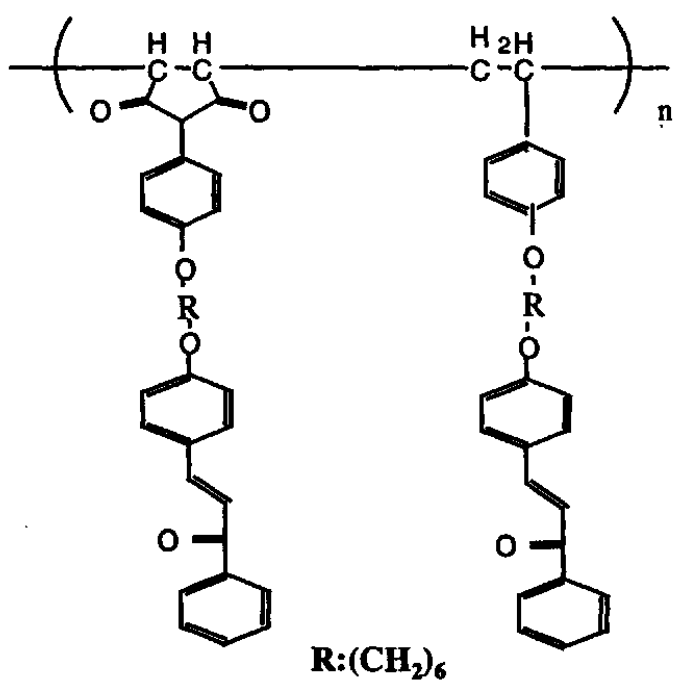

Fig. 9. Chemical structure of photo-alignment material, PMI-15.

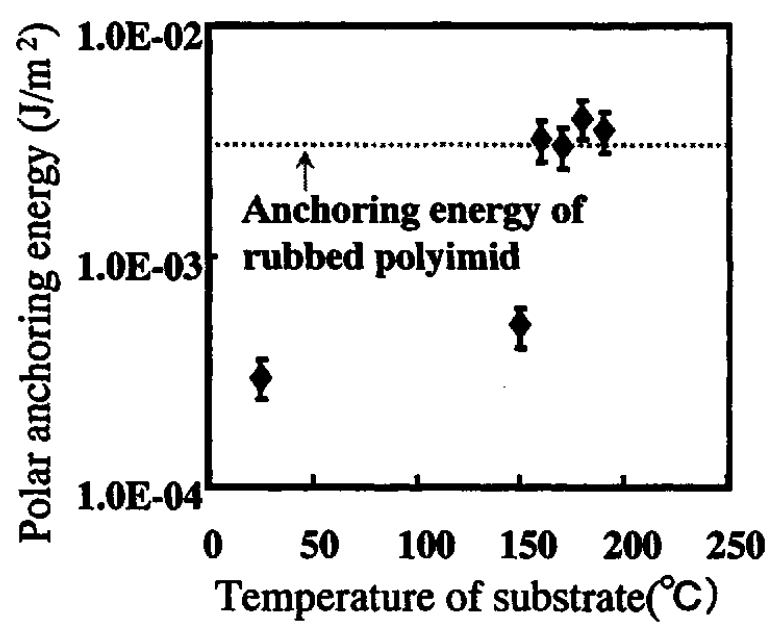

Fig. 10. Temperature dependence of polar anchoring energy of PMI-15.

By irradiation of linear polarized UV light on PMI-15 shown in Fig. 9, it shows good alignment characteristics of liquid crystals. Upon UV irradiation beyond $150{ }^{\circ} \mathrm{C}$ of substrate temperature, polar anchoring energy for liquid crystalline molecules is $10^{-3}$ order. It is almost same level in comparison with a rubbing alignment layer $^{8}$ (Fig.10). The ir absorption strength of 


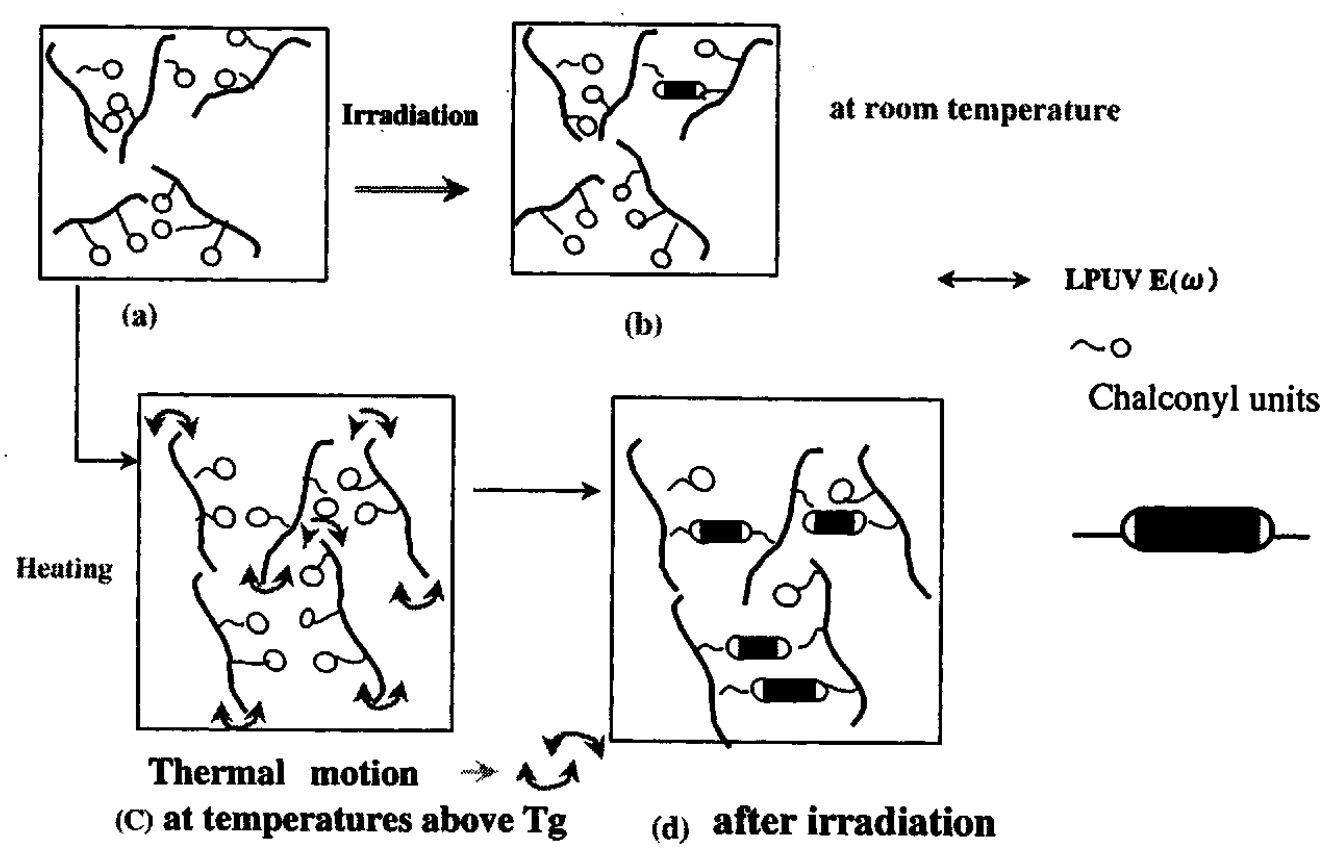

Fig.11. Schematic illustration of photo-alignment mechanism by dimerization of chalconyl unit.

$\mathrm{C}=\mathrm{C}$ bond reduced over $150^{\circ} \mathrm{C}$. It suggests that the photo-dimerization is accelerated over $\mathrm{Tg}$ of polymer. Fig. 11 shows a schematic illustration of photo-dimerization of chalcoyl unit over and under $\mathrm{Tg}$ of polymer.

PMI series of materials show not only good alignment characteristics but also pretilt angle generation by one step UV irradiation. Incorporating a long side chain to PMI series, they show high pretilt angle over 10deg depending on the incident angle of linear polarized UV'). And PMI series show good electrical property of high voltage holding ratio as a LCD cell. Residual DC is still worse in comparison with conventional LCD. However, this result shows a possibility to apply a photo-alignment material to industrial production of LCD near future.

\section{Conclusion}

In the LCD production process, many photo-polymers are used and contribute to improve LCD performance. LCD market is growing up rapidly, so that a newly developed photo-polymer is also required and expected. Photo-polymer science contributes expanding the market of LCD continuously.

\section{Acknowledgments}

Photo-alignment materials work was performed under management of ASET in the MITI's R\&D Program supported by NEDO.

\section{References}

1) H. Kawakami, Flat-Panel Display2001,96.

2) M. Endo, MonthlyDisplay, 5(7), 62(1999).

3) M. Yamashita, EKISHO, 4(1), 43(2000).

4) T. Nishio et al., IDW'99, 359(1999).

5) K. Yano, Flat-Panel Display2000,120.

6) A. Takeda, EKISHO, 3(2), 117(1999).

7) S. Nakata et al., SID'99, Digest, 512(1999).

8) M. Kimura et al., IDW'00, 53(2000).

9) S. Nakata et al., IDW'00, 1161(2000). 